\title{
VALORACIÓN DE LAS CARACTERÍSTICAS FISICOQUÍMICAS DE AVENA INSTANTÁNEA Y CREMA DE ARROZ
}

\section{RATING PHYSICO-CHEMICAL CHARACTERISTICS AND CREAM INSTANT OATMEAL RICE}

\author{
Quintero Cindy J. A. ${ }^{1}$, *Maldonado O. Yohanna. ${ }^{1}$, Caballero P. Luz Alba ${ }^{1}$, Rivera María \\ Esther ${ }^{2}$. \\ ${ }^{1}$ Facultad de Ingenierías y Arquitectura. Departamento de Alimentos. Grupo Investigaciones GIBA. Universidad de \\ Pamplona. Pamplona. Colombia. \\ ${ }^{2}$ Facultad de Ingenierías y Arquitectura. Departamento Civil y Ambiental. Grupo de Investigaciones Ambientales Agua, \\ Aire y Suelo (GIAAS). Universidad de Pamplona. Pamplona. Colombia \\ Correos electrónicos:cindyj0613@hotmail.com, *iohamalob@gmail.com, \\ luzcaballero@unipamplona.edu.co,maes@unipamplona.edu.co.
}

Recibido 21 de Agosto 2015; aceptado 30 de Octubre de 2015

\section{RESUMEN}

El objetivo del trabajo fue valorar las características fisicoquímicas de avena instantánea sabor fresa y crema de arroz sabor arequipe con el fin de definir sus parámetros de control de calidad, escogiendo dos productos comercializados como muestras patrón (Fortiavena, Quaker y Arroz Poli). Se definieron seis formulaciones para avena instantánea y cinco para crema de arroz. Se realizaron las siguientes pruebas fisicoquímicas por triplicado: cenizas, humedad, capacidad de hidratación (CH), índice de solubilidad en agua (ISA), distribución del tamaño de la partícula y sedimentación. Los resultados obtenidos fueron tratados mediante un análisis de varianza (ANOVA) para determinar diferencias estadísticamente significativas entre las formulaciones frente a las muestras patrón, con un nivel de significancia del 0.05. Se encontró que las formulaciones 
óptimas para avena instantánea fueron 2 y 3, y para crema de arroz la 5, cumpliendo con los parámetros de calidad en las normas para avena y crema de arroz.

Palabras clave: avena instantánea, características fisicoquímicas, crema de arroz, harina.

\section{ABSTRACT}

The objective of the work was to value the physical-chemical characteristics of oat instantaneous flavor strawberry and it cremates of rice flavor arequipe with the purpose of defining its parameters of control of quality, choosing two products marketed as samples pattern (Fortiavena, Quaker and Rice Poli). They were defined six formulations for instantaneous oat and five for cream of rice. They were carried out the following ones you prove physical-chemical had triplicated: ashy, humidity, hydrate capacity $(\mathrm{CH})$, index of solubility in water (ISA), distribution of the size of the particle and sedimentation. The obtained results were treated by means of a variance analysis (ANOVA) to determine differences statistically significant among the formulations in front of the samples pattern, with a level of significant of the 0.05 . it was found that the good formulations for instantaneous oat were 2 and 3, and for cream of rice the 5, fulfilling the parameters of quality in the norms for oat and it cremates of rice.

*Johana Maldonado Obando. $E$ mail: johamaldonadob@hotmail.com

Keywords: it trenches instantaneous, physical-chemical characteristics, it cremates of rice, flour.

\section{INTRODUCCIÓN}

La avena es un cultivo herbáceo anual que actualmente crece en todo el mundo y se utiliza principalmente para alimentación humana y animal. Las harinas de avena desarrollan rápidamente aromas y sabores de carácter amargo asociados a la acción 
de la lipasa, lipoxigenasa, y peroxidasa. Las partículas grandes de salvado y los tiempos cortos de fermentación limitan la degradación de los beta-glucanos durante la cocción. Los productos comerciales de avena más corrientes incluyen avena laminada (copos), avena desnuda cortada, avena instantánea o rápida, copos de avena para bebés, salvado de avena, y harina de avena.

Según la NTC 2160 (Productos de Molinería. Harina de Avena Precocida para consumo humano), la harina de avena es el producto obtenido de la molienda y tamizado de la avena en hojuelas. La harina de avena precocida debe ser fabricada a partir de avena en hojuelas o de granos de avena precocidos libres de signos de infestación o contaminación por roedores.

Otro cereal de suma importancia es el arroz, el cual pertenece al género Oryzay se cultivan dos especies, aunque existen 22 especies silvestres. El arroz proveniente del campo es el entero, conocido mundialmente por paddy o arroz cáscara; contiene cáscara externa fibrosa y no comestible llamada cascarilla (glumas y glumillas). El arroz, además de consumirse como grano entero, puede ser ampliamente usado en la fabricación de harinas, la molienda del arroz incluye el descascarillado, la eliminación del salvado y finalmente la separación de los granos partidos y dañados. (Gómez et al., 2006). La molienda de arroz generalmente origina desde un 4 a $40 \%$ de granos partidos, dependiendo de la calidad del grano de arroz y del equipo de molienda usado. Los granos partidos se separan de los granos enteros para obtener arroz de mesa con la calidad adecuada. Los criterios de la calidad más importantes del arroz son el rendimiento de molienda, la apariencia del grano crudo, el comportamiento en cocción y la textura y aroma del grano cocido (Juliano 1984, León et al., 2002).

El arroz como el resto de los cereales, es rico en hidratos de carbono complejos, es fuente de proteínas y minerales y no contiene colesterol. Los hidratos de carbono son los constituyentes más abundantes en el arroz, con un contenido aproximado de almidón del $80 \%$ (14\% humedad). almidón de arroz es un polímero de glucosas, compuesto por amilosa y amilopectina en diferentes proporciones según la variedad. El almidón determina las propiedades y la funcionalidad de los granos de arroz, y éstas son dependientes en gran parte de la relación amilosa/Amilopectina (Fitzgerald, 2004).

El arroz es uno de los cereales más pobres en proteínas (algunas variedades mejoradas pueden sin embargo, contener 
$14 \mathrm{~g}$ en $100 \mathrm{~g})$. El arroz además de consumirse como grano entero puede ser ampliamente usado en la fabricación de harinas. Sin embargo, muy poca producción de arroz se dedica a la elaboración de harinas y otros subproductos, la molienda del arroz incluye el descascarillado, la eliminación del salvado y finalmente la separación de los granos partidos y dañados. (Rosell, 2007). La molienda seca consiste en la trituración y reducción de tamaño de los granos partidos. Esta harina se usa para alimentos infantiles, productos extruídos y diversos productos de panificación. Las harinas de arroz tienen la misma composición química que los granos de donde proceden. Por tanto las propiedades funcionales de las harinas de arroz dependen de la variedad, condiciones medioambientales y métodos de molienda. Las harinas se distinguen por el contenido de amilosa, el cual determina la temperatura de gelatinización y las propiedades viscoelásticas (Fan et al., 1998, Singh et al., 2000, Meadows 2002, Saif et al., 2003).
Harinas provenientes de arroces cerosos que contienen poca amilosa (menos de $0,56 \%$ ) gelatinizan a temperaturas más bajas y presentan menor retrogradación, y por eso son resistentes a la sinéresis durante el almacenamiento y la congelación (Juliano, 1984). Si se utilizan harinas procedentes de variedades con diferente contenido de amilosa (bajo, medio y alto amilosa) se podría ampliar el espectro de uso de estas harinas, proporcionando así alternativas a la industria de alimentos y especialmente a la de nuevos productos.

La harina de arroz se obtiene mayoritariamente de los granos de arroz pulidos, aunque en ocasiones se obtienen del arroz integral. La harina procedente de granos integrales posee mayor temperatura de gelatinización (Normand y Marshall, 1989) y tiene un mayor contenido de fibra y vitaminas, debido a su mayor abundancia en las partes externas del grano.

\section{MATERIALES Y MÉTODOS}

Se realizaron las siguientes pruebas fisicoquímicas para determinar la calidad de las harinas. Los análisis se realizaron por triplicado para cada una de las muestras de harinas obtenidas en la empresa y tres tipos de harinas comerciales tomadas como patrón.

Evaluación fisicoquímica de la Avena Instantánea sabor Fresa y Crema de Arroz sabor Arequipe. 
Determinación de Cenizas. Se pesó con precisión $3 \mathrm{~g}$ de muestra, utilizando una balanza analítica marca KERN AB5 la cual fue calcinada hasta que se obtuvo un residuo carbonoso en un crisol previamente incinerado y tarado, teniendo cuidado de que la combustión no fuera demasiado rápida, de manera que no hubiesen pérdidas de materia sólida por proyección. Posteriormente se introdujo el crisol en la mufla marca NEYVULCAN 3-550 a una temperatura de $550 \pm 10{ }^{\circ} \mathrm{C}$, hasta combustión completa de la sustancia (cenizas blancas o grises). Luego se enfrió a temperatura ambiente en un desecador y se registró el peso. Finalmente se determinó el porcentaje de cenizas mediante la siguiente fórmula:

$$
\% \text { Ceniza }=\frac{P 1-P 2}{P} * 100
$$

Siendo:

$\mathrm{P}_{1}=$ Peso, en gramos, del crisol con las cenizas.

$\mathrm{P}_{2}=$ Peso, en gramos, del crisol vacío.

$\mathrm{P}=\mathrm{Peso}$, en gramos, de la muestra.

Capacidad de Hidratación (CH). Se realizó de acuerdo con la metodología descrita por Leach et al., (1959), con algunas modificaciones que consistieron en usar tres temperaturas $\left(60,70\right.$ y $\left.80 \pm 1{ }^{\circ} \mathrm{C}\right)$. Cada muestra de harina de $0.74 \mathrm{~g}$ (base seca) se colocó en tubos de propileno de $15 \mathrm{ml}$ previamente tarados y se adicionó $12 \mathrm{ml}$ de agua destilada; luego se colocaron en baño
María en planchas de calentamiento, con agitación, marca Schott Instruments. La suspensión se mantuvo en agitación y temperatura constantes durante $30 \mathrm{~min}$; posteriormente se centrifugó a 3000 RPM durante $10 \mathrm{~min}$, en una centrifuga marca Becton Dickinson Compact II. sobrenadante se separó cuidadosamente y se registró el peso del residuo sólido. El sobrenadante se evaporó en estufa a una temperatura de $105 \pm 1{ }^{\circ} \mathrm{C}$, en la mufla NEYVULCAN 3-550, hasta peso constante y luego se pesó con ayuda de la balanza analítica KERN AB5. La capacidad de hidratación $(\mathrm{CH})$ se calculó mediante la relación del peso del residuo de centrifugación y el peso seco de la muestra en cada temperatura probada.

Índice de Solubilidad en Agua (ISA). Esta variable se midió de acuerdo con la metodología descrita por Anderson et al., (1969), empleando para el cálculo el mismo criterio descrito para la capacidad de hidratación.

Distribución del Tamaño de Partícula. Se pesó una muestra de $50 \mathrm{~g}$ de cada una de las harinas en una balanza analítica marca KERN AB5, se colocó en un tamiz rotatorio, dotado de 8 mallas que van desde 0,063 $\mathrm{mm}$ hasta $2 \mathrm{~mm}$ de luz de malla, después se sometió a agitación, con una potencia de 9 y 9 ciclos a un tiempo de 20 minutos, el tamaño de la partícula se determinó con 
base al peso de las fracciones retenidas en las diferentes mallas.

Prueba de Sedimentación. Se colocaron 4 $\mathrm{g}$ de muestra en una probeta graduada y con tapón a las cuales se añadieron $50 \mathrm{ml}$ de solución hidratada con azul de bromofenol. Posteriormente se mezcló y agitando doce veces de izquierda a derecha durante 5 segundos. A continuación se dejó suspender totalmente la harina y se continuó agitando por cinco minutos más y se adicionaron $25 \mathrm{ml}$ del reactivo de prueba y se agitó por 10 minutos. Finalmente se dejó reposar cinco minutos y se tomó la lectura del volumen de la fase sólida.

Humedad. La humedad se determinó mediante el método de termo-balanza XM 60 PRECISA. Se tomó una muestra de $3 \mathrm{~g}$ de cada una de las muestras, posteriormente se colocaron en el platillo y se graduó la temperatura a $110 \pm 1{ }^{\circ} \mathrm{C}$ con un tiempo de 30 minutos registrando la humedad final.

Análisis Estadístico. Se realizó un análisis de varianza (ANOVA) con intervalo de confianza del $95 \%$, para comprobar la existencia de diferencias estadísticamente significativas entre las frecuencias de respuestas para cada producto, para lo cual, se empleó el paquete estadístico SPSS versión 17.

\section{RESULTADOS Y DISCUSIÓN}

Determinación de cenizas. Todas las formulaciones de avena presentaron un porcentaje de cenizas de $1.7 \%$ y la muestra patrón $2 \%$, este valor se encuentra dentro del rango permitido por la NTC 2160. Se evidencia que existe una diferencia del 0.3 $\%$ entre la muestra patrón y las formulaciones de la empresa, lo cual puede presentarse debido a que en la composición química de la avena utilizada en la empresa no es la misma en todos los casos y se desconoce la variedad de la formulación patrón. Las formulaciones de crema de arroz presentaron un porcentaje medio de cenizas de $0.8 \%$ y la patrón $0.6 \%$, ambos resultados son menores que el porcentaje de ceniza que presenta el grano completo, teniendo en cuenta que el salvado constituye el 10 - $15 \%$ (Champagne, 2004). Es importante destacar que el contenido de minerales que presenta la crema de arroz de la empresa es mayor que el de los productos de la competencia, posiblemente debido a la adición de aceite mineral a la materia prima. 
Capacidad de hidratación (CH). Teniendo en cuenta las formulaciones se realizó el Análisis de Varianza (ANOVA) de las pruebas de calidad realizadas a los diferentes tipos de harinas en avena Instantánea sabor fresa y crema de arroz sabor arequipe.

Tabla 1. Resultados de los análisis de Varianza (ANOVA) de las pruebas de calidad para avena instantánea.

\begin{tabular}{|c|c|c|}
\hline Fuente de Variación & $\mathbf{F}$ & Sig. (P-valor) \\
\hline HUMEDAD & 100.637 & $0.000^{\star}$ \\
\hline $\begin{array}{c}\text { TAMAÑO_PARTICU } \\
\text { LA }\end{array}$ & 191.757 & $0.000^{\star}$ \\
\hline $\mathrm{CH} \_60^{\circ} \mathrm{C}$ & 1.814 & 0.153 \\
\hline $\mathrm{CH} \_70^{\circ} \mathrm{C}$ & 2.103 & 0.103 \\
\hline $\mathrm{CH} \_80^{\circ} \mathrm{C}$ & 11.061 & $0.000^{\star}$ \\
\hline ISA_60 ${ }^{\circ} \mathrm{C}$ & 43.703 & $0.000^{\star}$ \\
\hline ISA_70 ${ }^{\circ} \mathrm{C}$ & 62.706 & $0.000^{\star}$ \\
\hline ISA_80 ${ }^{\circ} \mathrm{C}$ & 12.411 & $0.000^{\star}$ \\
\hline
\end{tabular}
para un nivel de significancia $(\alpha)=0.05$.

De la tabla 1, se determinó que existen diferencias estadísticamente significativas para un nivel de significancia $(\alpha)=0.05$ en avena instantánea en las pruebas de humedad, tamaño de partícula, $\mathrm{CH}$ a temperatura de $80 \pm 1^{\circ} \mathrm{C}$ e ISA en todas las temperaturas. Por lo tanto es necesario aplicar la Prueba Post hoc de Tukey para cada parámetro de calidad en avena instantánea sabor fresa.

En crema de arroz se realizaron pruebas de Calidad a las formulaciones (1 a 5) y un patrón (Arroz Poli), el tratamiento de los resultados se llevó a cabo mediante el análisis de varianzas (ANOVA) para un nivel de significancia $(\alpha)$ de 0.05 (tabla 2).

Tabla 2. Resultados de los análisis de Varianza (ANOVA) de las pruebas de calidad para crema de arroz.

\begin{tabular}{|c|c|c|c|}
\hline \multicolumn{2}{|c|}{ Fuente de Variación } & F & $\begin{array}{c}\text { Sig. (p- } \\
\text { valor) }\end{array}$ \\
\hline HUMEDAD & Inter-grupos & 88.733 & $\mathbf{0 . 0 0 0 ^ { * }}$ \\
\hline $\begin{array}{c}\text { TAMAÑO_- } \\
\text { PARTICULA }\end{array}$ & Inter-grupos & 45.893 & $\mathbf{0 . 0 0 0 ^ { * }}$ \\
\hline CH_60 & Inter-grupos & 2.439 & 0.096 \\
\hline CH_70 & Inter-grupos & 1.169 & 0.379 \\
\hline CH_80 & Inter-grupos & 35.028 & $\mathbf{0 . 0 0 0 ^ { * }}$ \\
\hline ISA_60 & Inter-grupos & 1.593 & 0.235 \\
\hline ISA_70 & Inter-grupos & 3.424 & $\mathbf{0 . 0 3 7 ^ { * }}$ \\
\hline ISA_80 & Inter-grupos & 0.821 & 0.558 \\
\hline
\end{tabular}

*Existen diferencias estadísticamente significativas para un nivel de significancia $(\alpha)=0.05$.

De la tabla anterior, se evidencia con el análisis de varianzas (ANOVA) que la crema de arroz presenta diferencias estadísticamente significativas para un nivel de significancia $(\alpha)$ de 0.05 en las pruebas de humedad, tamaño de partícula y $\mathrm{CH}$ a temperatura de $70{ }^{\circ} \mathrm{C}$. Por lo tanto es necesario aplicar la Prueba Post hoc de Tukey para cada parámetro de calidad en crema de arroz sabor arequipe.

La Prueba Post hoc de Tukey de la capacidad de hidratación $(\mathrm{CH})$ a diferentes temperaturas se muestra en la tabla 3 con el fin de encontrar las diferencias entre las 
formulaciones de avena instantánea planteadas.

Tabla 3. Prueba Post Hoc de Tukey de la capacidad de hidratación para avena instantánea sabor fresa.

\begin{tabular}{|c|c|c|}
\hline $\begin{array}{l}\text { Parámetro } \\
\text { de calidad }\end{array}$ & MUESTRAS & $\begin{array}{c}\text { MEDIA } \pm \\
\text { DESVIACIÓN } \\
\text { ESTANDAR }\end{array}$ \\
\hline \multirow{8}{*}{ CH_60 } & Formulacion_1 & $4.5651 \pm 0.16315^{a}$ \\
\hline & Formulacion_2 & $3.9784 \pm 0.05395^{a}$ \\
\hline & Formulacion_3 & $4.1706 \pm 0.06655^{a}$ \\
\hline & Formulacion_4 & $4.2835 \pm 0.2968^{a}$ \\
\hline & Formulacion_5 & $4.0581 \pm 0.55315^{a}$ \\
\hline & Gran_Señora (GS) & $3.5512 \pm 0.8068^{a}$ \\
\hline & Fortiavena & $4.1244 \pm 0.0877^{a}$ \\
\hline & Quaker & $4.3959 \pm 0.37035^{a}$ \\
\hline \multirow{8}{*}{ CH_70 } & Formulacion_1 & $4.8067 \pm 0.09355^{a}$ \\
\hline & Formulacion_2 & $4.2984 \pm 0.64055^{a}$ \\
\hline & Formulacion_3 & $4.2128 \pm 0.6299^{a}$ \\
\hline & Formulacion_4 & $4.6417 \pm 0.2536^{a}$ \\
\hline & Formulacion_5 & $\pm 0.257^{a}$ \\
\hline & Gran_Señora (GS) & $4.1418 \pm 0.7041^{\mathrm{a}}$ \\
\hline & Fortiavena & $4.4128 \pm 0.05395^{\mathrm{a}}$ \\
\hline & Quaker & $5.2148 \pm 0.14275^{a}$ \\
\hline \multirow{8}{*}{ CH_80 } & Formulacion_1 & $4.7378 \pm 0.15585^{a}$ \\
\hline & Formulacion_2 & $4.4875 \pm 0.2334^{\mathrm{a}}$ \\
\hline & Formulacion_3 & $4.3193 \pm 0.2976^{a}$ \\
\hline & Formulacion_4 & $4.2195 \pm 0.26675^{a}$ \\
\hline & Formulacion_5 & $4.1157 \pm 0.3664^{a}$ \\
\hline & Gran_Señora (GS) & $\pm 0.8106^{a}$ \\
\hline & Fortiavena & $\pm 0.0524^{a}$ \\
\hline & Quaker & $6.1363 \pm 0.2419^{b}$ \\
\hline
\end{tabular}

Letras iguales no existen diferencias estadísticamente significativas.

Se realizó un diagrama de barras representando las diferencias entre las diferentes formulaciones de avena instantánea sabor fresa. (Ver figura 1)

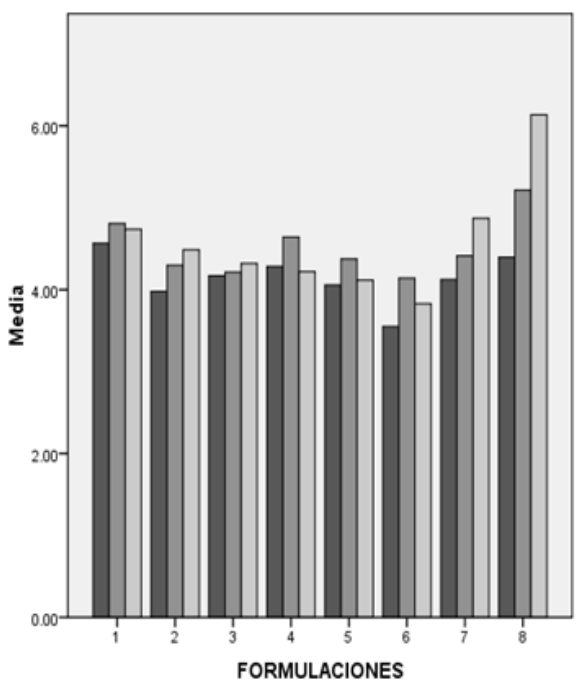

Figura 1. Diagrama de barras para la capacidad de hidratación en avena instantánea sabor fresa.

De la figura 1 y la tabla 3 , se infiere que $\mathrm{CH}$ (capacidad de hidratación) a temperaturas de 60 y $70 \pm 1{ }^{\circ} \mathrm{C}$ no existen diferencias estadísticamente significativas, mientras que a temperatura de $80 \pm 1{ }^{\circ} \mathrm{C}$ la formulación 8 (Quaker) difiere de todas las demás formulaciones, ya que posee mayor capacidad de hidratación que las demás. Estas diferencias se pueden atribuir a variaciones en el grado de cocimiento o ausencia del proceso de precocidos en los productos de la empresa, o bien a diferencias en la dureza del grano usado como materia prima. (Flóres-Farías et al., 2002; Gómez et al., 1991). Lo que indica que si la empresa tiene como formulación patrón Fortiavena su $\mathrm{CH}$ es muy similar en las formulaciones (1 a 5), aunque la formulación de la empresa es menor comparada con las demás, pero si desea 
que su patrón sea Quaker debe mejorar su $\mathrm{CH}$. A continuación se presentan los resultados de la Prueba Post Hoc de Tukey de la capacidad de hidratación $(\mathrm{CH})$ a diferentes temperaturas (tabla 4), con el fin de encontrar las diferencias entre las formulaciones (1 a 5) de crema de arroz sabor arequipe planteadas y un patrón (Arroz Poli).

Tabla 4. Resultados de la prueba Post Hoc de Tukey de la capacidad de hidratación para crema de arroz sabor arequipe.

\begin{tabular}{|c|c|c|}
\hline $\begin{array}{l}\text { Parámetro } \\
\text { de calidad }\end{array}$ & MUESTRAS & $\begin{array}{c}\text { MEDIA } \pm \\
\text { DESVIACIÓN } \\
\text { ESTANDAR }\end{array}$ \\
\hline \multirow{6}{*}{ CH_60 } & Formulacion_1 & $2.5758 \pm 0.099^{a}$ \\
\hline & Formulacion_2 & $2.3789 \pm 0.346^{a}$ \\
\hline & Formulacion_3 & $2.0737 \pm 0.213^{a}$ \\
\hline & Formulacion_4 & $2.6002 \pm 0.236^{a}$ \\
\hline & Formulacion_5 & $2.4894 \pm 0.486^{a}$ \\
\hline & Arroz_Poli & $2.8533 \pm 0.176^{a}$ \\
\hline \multirow{6}{*}{ CH_70 } & Formulacion_1 & $5.5694 \pm 0.243^{a}$ \\
\hline & Formulacion_2 & $5.2733 \pm 0.817^{a}$ \\
\hline & Formulacion_3 & $5.5906 \pm 0.744^{a}$ \\
\hline & Formulacion_4 & $5.3782 \pm 0.121^{a}$ \\
\hline & Formulacion_5 & $5.5483 \pm 0.069^{a}$ \\
\hline & Arroz_Poli & $6.1200 \pm 0.131^{a}$ \\
\hline \multirow{6}{*}{ CH_80 } & Formulacion_1 & $5.4247 \pm 0.649^{a}$ \\
\hline & Formulacion_2 & $5.7067 \pm 0.110^{a}$ \\
\hline & Formulacion_3 & $5.4743 \pm 0.180^{\circ}$ \\
\hline & Formulacion_4 & $5.6393 \pm 0.365^{a}$ \\
\hline & Formulacion_5 & $5.8484 \pm 0.128^{a}$ \\
\hline & Arroz_Poli & $8.6667 \pm 0.437^{b}$ \\
\hline
\end{tabular}

Letras iguales no existen diferencias estadísticamente significativas.

Se realizó el diagrama de barras entre las formulaciones y la capacidad de hidratación en crema arroz sabor arequipe (Ver figura 2), observando la variación de $\mathrm{CH}$ con respecto a la temperatura, para cada formulación. La capacidad de hidratación se debe a la composición de la materia prima, la cantidad y el tipo de almidón, aunque los hidratos de carbono son los constituyentes más abundantes en el arroz, con un contenido aproximado de almidón del $80 \%$ (14 \% humedad). La figura 2 muestra que la capacidad de hidratación en crema de arroz presenta el mismo comportamiento que en avena instantánea en todas sus formulaciones, aunque para este parámetro las formulaciones (1 a 5) de la empresa son menores que el patrón Arroz Poli.
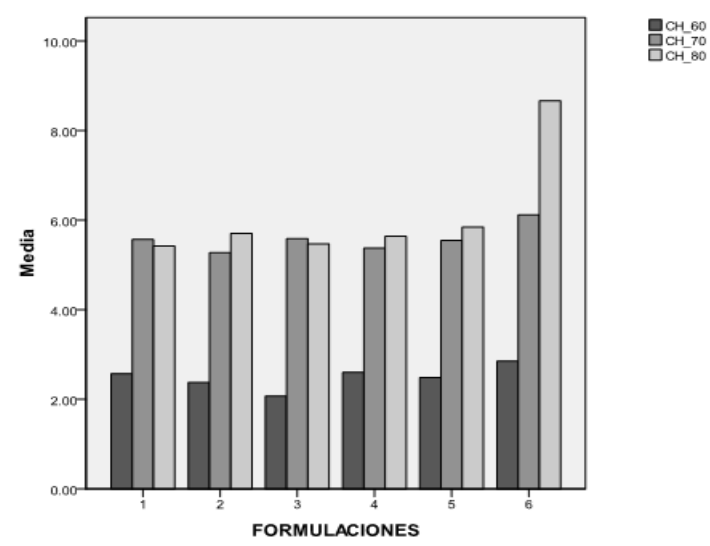

Figura 2. Diagrama de barras para la capacidad de hidratación en crema de arroz sabor arequipe.

La materia prima utilizada para la elaboración de avena instantánea donde se utiliza completa y se aprovecha el contenido de fibra presente en la cascarilla; por tanto a 
(a)LIMENTECH CIENCIA Y TECNOLOGÍA ALIMENTARIA ISSN 1692-7125. Volumen 13, No. 2, p. 205-220, año 2015 Facultad de Ingenierías y Arquitectura Universidad de Pamplona

mayor cantidad de fibra, mayor $\mathrm{CH}$ debido a la presencia de hemicelulosa y a la estructura amorfa de la misma; (Sánchez, 2005).

Índice de Solubilidad de Agua (ISA). En el análisis de varianza ANOVA, se encontraron diferencias estadísticamente significativas para los índices de solubilidad de agua en avena instantánea sabor fresa y crema de arroz sabor arequipe, por lo tanto es necesario aplicar la Prueba Post hoc de Tukey (ver tablas 5 y 6) para encontrar las diferencias entre las formulaciones.

Tabla 5. Resultados de la Prueba Post Hoc de Tukey de índice de solubilidad de agua para avena instantánea sabor fresa.

\begin{tabular}{|c|c|cc|}
\hline $\begin{array}{l}\text { Parámetro } \\
\text { de calidad }\end{array}$ & MUESTRAS & \multicolumn{2}{|c|}{$\begin{array}{c}\text { MEDIA } \\
\text { DESVIACION } \\
\text { ESTANDAR }\end{array}$} \\
\hline \multirow{4}{*}{ ISA_60 } & Formulacion_1 & 61.14 & $\pm 0.250^{\mathrm{a}}$ \\
\cline { 2 - 4 } & Formulacion_2 & 60.06 & $\pm 0.080^{\mathrm{a}}$ \\
\cline { 2 - 4 } & Formulacion_3 & 61.32 & $\pm 1.270^{\mathrm{a}}$ \\
\cline { 2 - 4 } & Formulacion_4 & 61.55 & $\pm 0.780^{\mathrm{a}}$ \\
\cline { 2 - 4 } & Formulacion_5 & 61.88 & $\pm 1.450^{\mathrm{a}}$ \\
\cline { 2 - 4 } & Gran_Señora (GS) & 61.98 & $\pm 2.200^{\mathrm{a}}$ \\
\cline { 2 - 4 } & Fortiavena & 65.30 & $\pm 0.377^{\mathrm{b}}$ \\
\cline { 2 - 4 } & Quaker & 72.95 & $\pm 0.512^{\mathrm{b}}$ \\
\hline \multirow{5}{*}{ ISA_70 } & Formulacion_1 & 68.75 & $\pm 0.255^{\mathrm{a}}$ \\
\cline { 2 - 4 } & Formulacion_2 & 69.27 & $\pm 0.115^{\mathrm{a}}$ \\
\cline { 2 - 4 } & Formulacion_3 & 69.01 & $\pm 0.630^{\mathrm{a}}$ \\
\cline { 2 - 4 } & Formulacion_4 & 68.88 & $\pm 0.955^{\mathrm{a}}$ \\
\cline { 2 - 4 } & Formulacion_5 & 69.18 & $\pm 0.465^{\mathrm{a}}$ \\
\cline { 2 - 4 } & Gran_Señora (GS) & 69.08 & $\pm 0.795^{\mathrm{a}}$ \\
\cline { 2 - 4 } & Fortiavena & 73.76 & $\pm 0.090^{\mathrm{bc}}$ \\
\cline { 2 - 4 } & Quaker & 74.69 & $\pm 0.065^{\mathrm{bd}}$ \\
\hline \multirow{5}{*}{ ISA_80 } & Formulacion_1 & 72.79 & $\pm 1.220^{\mathrm{a}}$ \\
\cline { 2 - 4 } & Formulacion_2 & 73.24 & $\pm 0.605^{\mathrm{a}}$ \\
\cline { 2 - 4 } & Formulacion_3 & 72.83 & $\pm 0.330^{\mathrm{a}}$ \\
\cline { 2 - 4 } & Formulacion_4 & 72.95 & $\pm 0.325^{\mathrm{a}}$ \\
\cline { 2 - 4 } & Formulacion_5 & 74.03 & $\pm 0.350^{\mathrm{a}}$ \\
\cline { 2 - 4 } & Gran_Señora (GS) & 72.55 & $\pm 0.985^{\mathrm{a}}$ \\
\cline { 2 - 4 } & Fortiavena & 74.53 & $\pm 0.450^{\mathrm{a}}$ \\
\cline { 2 - 4 } & Quaker & 77.33 & $\pm 1.225^{\mathrm{b}}$ \\
\hline
\end{tabular}

Letras iguales no existen diferencias estadísticamente significativas

Se realizó un diagrama de barras (figura 3), con el fin de evidenciar mejor variación del índice de solubilidad en agua (ISA) con respecto a la temperatura para las formulaciones (1 a 6) de avena instantánea y dos muestras patrón.

De la figura 3, se infiere que ISA (índice de solubilidad de agua) a temperaturas de 60 y $70 \pm 1{ }^{\circ} \mathrm{C}$ no existen diferencias estadísticamente significativas entre las formulaciones y la muestras control (Quaker y Fortiavena), mientras que a temperatura de $80 \pm 1{ }^{\circ} \mathrm{C}$ la formulación 8 (avena Quaker) difiere de todas las demás formulaciones.

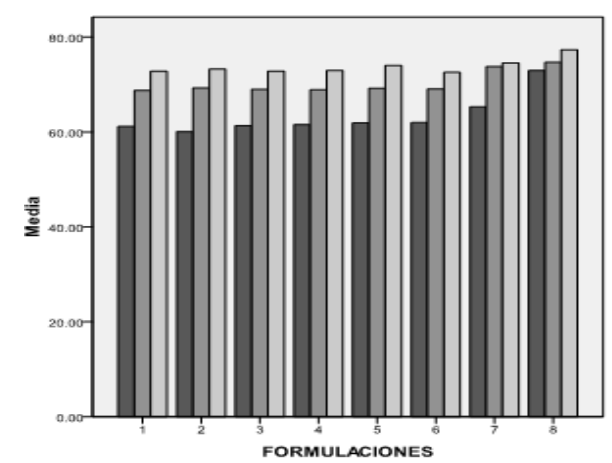

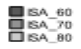

Figura 3. Índice de solubilidad en agua para avena instantánea.

A continuación se presentan los resultados de la Prueba Post Hoc de Tukey de índice de solubilidad de agua (ISA) para crema de arroz sabor arequipe. (tabla 6). 
Tabla 6. Resultados de la Prueba Post Hoc de Tukey de índice de solubilidad de agua (ISA) para crema de arroz sabor arequipe.

\begin{tabular}{|c|c|c|c|}
\hline $\begin{array}{l}\text { Parámetro } \\
\text { de color }\end{array}$ & MUESTRAS & \multicolumn{2}{|c|}{$\begin{array}{c}\text { MEDIA } \pm \text { DESVIACION } \\
\text { ESTANDAR }\end{array}$} \\
\hline \multirow{6}{*}{ ISA_60 } & Formulacion_1 & 48.970 & $\pm 1.36^{a}$ \\
\hline & Formulacion_2 & 50.603 & $\pm 1.045^{a}$ \\
\hline & Formulacion_3 & 50.703 & $\pm 0.115^{a}$ \\
\hline & Formulacion_4 & 48.923 & $\pm 1.605^{a}$ \\
\hline & Formulacion_5 & 50.320 & $\pm 1.42^{a}$ \\
\hline & Arroz_Poli & 50.440 & $\pm 0.28^{a}$ \\
\hline \multirow{6}{*}{ ISA_70 } & Formulacion_1 & 53.053 & $\pm 0.725^{a}$ \\
\hline & Formulacion_2 & 55.303 & $\pm 0.545^{a}$ \\
\hline & Formulacion_3 & 54.15 & $\pm 1.23^{\mathrm{a}}$ \\
\hline & Formulacion_4 & 54.99 & $\pm 1.515^{a}$ \\
\hline & Formulacion_5 & 54.57 & $\pm 0.90^{\mathrm{a}}$ \\
\hline & Arroz_Poli & 56.05 & $\pm 0.367^{b}$ \\
\hline \multirow{6}{*}{ ISA_80 } & Formulacion_1 & 77.33 & $\pm 0.18^{a}$ \\
\hline & Formulacion_2 & 74.81 & $\pm 0.67^{a}$ \\
\hline & Formulacion_3 & 77.04 & $\pm 0.26^{a}$ \\
\hline & Formulacion_4 & 76.0067 & $\pm 0.05508^{a}$ \\
\hline & Formulacion_5 & 75.84 & $\pm 2.5^{\mathrm{a}}$ \\
\hline & Arroz_Poli & 55.3867 & $\pm 39.94702^{a}$ \\
\hline
\end{tabular}

Letras iguales no existen diferencias estadísticamente significativas.

Se realizó un diagrama de barras (figura 4), con el fin de evidenciar mejor la variación del índice de solubilidad en agua (ISA) con respecto a la temperatura para las formulaciones ( 1 a 5 ) de crema de arroz y patrón.

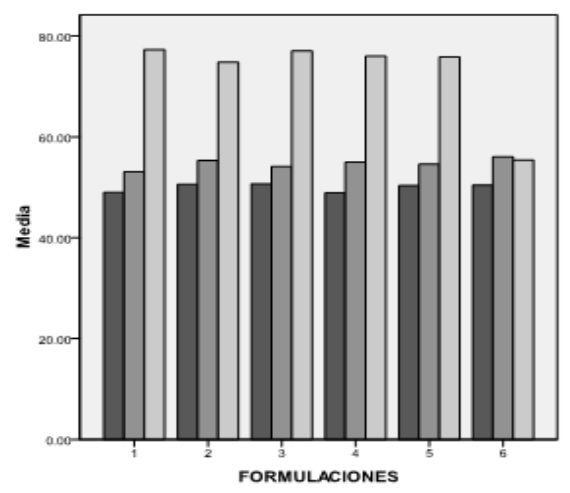

Figura 4. Índice de solubilidad en agua (ISA) para crema de arroz.
De la figura 4, se infiere que ISA (índice de solubilidad de agua) a temperaturas de $60 \mathrm{y}$ $80 \pm 1{ }^{\circ} \mathrm{C}$ no existen diferencias estadísticamente significativas entre las formulaciones y la muestra control, mientras que a temperatura de $70 \pm 1^{\circ} \mathrm{C}$ la formulación 6 (Arroz Poli) difiere de todas las demás formulaciones. El índice de solubilidad y la capacidad de hidratación presentaron también una respuesta dependiente de la temperatura, pues el grado de gelatinización se relaciona positivamente con la cantidad de agua que hidrata a los gránulos de almidón (FloresFarías et al., 2002; Gómez et al., 1991). Este aspecto es de suma importancia en crema de arroz ya que la preparación del producto requiere cocción $\left(80 \pm 1^{\circ} \mathrm{C}\right)$, con el fin de que se produzca la gelatinización y se desarrollen las características de viscosidad.

Distribución del tamaño de partícula. En el análisis de varianza ANOVA, se encontraron diferencias estadísticamente significativas $(\alpha=0.05)$ para el tamaño de partícula en avena instantánea sabor fresa y crema de arroz sabor arequipe por lo tanto fue necesario aplicar la Prueba Post hoc de Tukey (ver tablas 7 y 8) para encontrar las diferencias entre las formulaciones.

Tabla 7. Prueba Post Hoc de Tukey de tamaño de partícula para avena instantánea sabor fresa. 
@-LIMENTECH CIENCIA Y TECNOLOGÍA ALIMENTARIA ISSN 1692-7125. Volumen 13, No. 2, p. 205-220, año 2015 Facultad de Ingenierías y Arquitectura Universidad de Pamplona

\begin{tabular}{|c|l|cc|}
\hline $\begin{array}{c}\text { Parámetro } \\
\text { de calidad }\end{array}$ & \multicolumn{1}{|c|}{ MUESTRAS } & \multicolumn{2}{|c|}{$\begin{array}{c}\text { MEDIA } \\
\text { DESVIACION } \\
\text { ESTANDAR }\end{array}$} \\
\hline \multirow{4}{*}{$\begin{array}{c}\text { Tamaño } \\
\text { de }\end{array}$} & Formulacion_1 & 1.2242 & $\pm 0.055^{\mathrm{a}}$ \\
\cline { 2 - 4 } Partícula & Formulacion_2 & 1.4427 & $\pm 0.0128^{\mathrm{bd}}$ \\
\cline { 2 - 4 } & Formulacion_3 & 1.5122 & $\pm 0.01865^{\mathrm{bd}}$ \\
\cline { 2 - 4 } & Formulacion_4 & 1.2311 & $\pm 0.0322^{\mathrm{a}}$ \\
\cline { 2 - 4 } & Formulacion_5 & 1.146 & $\pm 0.03345^{\mathrm{a}}$ \\
\cline { 2 - 4 } & Gran_Señora (GS) & 1.2214 & $\pm 0.01695^{\mathrm{a}}$ \\
\cline { 2 - 4 } & Fortiavena & 1.1942 & $\pm 0.02527^{\mathrm{a}}$ \\
\cline { 2 - 4 } & Quaker & 0.7351 & $\pm 0.01025^{\mathrm{bc}}$ \\
\hline
\end{tabular}

Letras iguales no existen diferencias estadísticamente significativas

De la tabla 7 se infiere que el tamaño de la partícula de las formulaciones 1, Gran Señora (GS) y Fortiavena difieren de las formulaciones 2, 3 y Quaker, así como la formulación Quaker difiere de todas las demás, así mismo se muestra que el tamaño de partícula de Quaker es menor que las demás formulaciones, evidenciando también menor capacidad de hidratación e índice de solubilidad en agua que las demás.

Tabla 8. Prueba Post Hoc de Tukey de tamaño de partícula para crema de arroz sabor arequipe.

\begin{tabular}{|c|l|rl|}
\hline $\begin{array}{c}\text { Parámetro } \\
\text { de calidad }\end{array}$ & \multicolumn{1}{|c|}{ MUESTRAS } & \multicolumn{2}{|c|}{$\begin{array}{c}\text { MEDIA } \pm \text { DESVIACION } \\
\text { ESTANDAR }\end{array}$} \\
\hline \multirow{4}{*}{$\begin{array}{c}\text { Tamaño } \\
\text { de } \\
\text { partícula }\end{array}$} & Formulacion_1 & 0.6237 & $\pm 0.1176^{a c}$ \\
\cline { 2 - 4 } & Formulacion_2 & 0.7794 & $\pm 0.0160^{\text {a }}$ \\
\cline { 2 - 4 } & Formulacion_3 & 0.6502 & $\pm 0.0127^{\text {ac }}$ \\
\cline { 2 - 4 } & Formulacion_4 & 1.3405 & $\pm 0.0329^{\text {b }}$ \\
\cline { 2 - 4 } & Formulacion_5 & 0.8504 & $\pm 0.1048^{\text {be }}$ \\
\cline { 2 - 4 } & Arroz_Poli & 0.6767 & $\pm 0.0451^{\text {bd }}$ \\
\hline
\end{tabular}

Letras iguales no existen diferencias estadísticamente significativas.

De la tabla 8 se determinó que el tamaño de la partícula de la formulación 4 difiere de todas las demás formulaciones, así como la formulaciones 1,3 y 4 difieren de la formulación 5. Aunque la formulación 4 presenta mayor tamaño de partícula, esta variación no se ve evidenciada en la capacidad de hidratación ni en el índice de solubilidad en agua debido a que este producto tiene un proceso de cocción durante su preparación. Por tanto se realiza un diagrama de dispersión donde se representa la distribución del tamaño de la partícula tanto para la avena instantánea como para la crema de arroz elaborados en la empresa. (Ver figura 5).

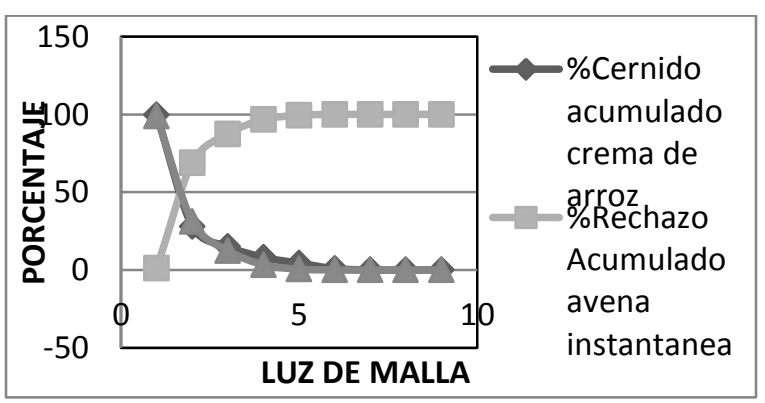

Figura 5. Tamaño de la Partícula para los productos crema de arroz y avena instantánea.

Como se observa en la figura 5 el tamaño de la partícula de la avena instantánea es 50 mm, cumpliendo con la NTC 2160.

Humedad. En el análisis de varianza ANOVA, se encontraron diferencias estadísticamente significativas para la humedad de las diferentes harinas, por lo que se aplicó la Prueba Post hoc de Tukey (ver tablas 9 y 10) para encontrar las diferencias entre las formulaciones. 
Tabla 9. Prueba Post Hoc de Tukey de humedad para avena instantánea sabor fresa.

\begin{tabular}{|c|c|c|c|}
\hline $\begin{array}{l}\text { Parámetro } \\
\text { de calidad }\end{array}$ & MUESTRAS & \multicolumn{2}{|c|}{$\begin{array}{c}\text { MEDIA } \pm \\
\text { DESVIACION } \\
\text { ESTANDAR }\end{array}$} \\
\hline \multirow{8}{*}{ Humedad } & Formulación 1 & 9.09 & $\pm 0.680^{a}$ \\
\hline & Formulación 2 & 10.02 & $\pm 0.420^{a}$ \\
\hline & Formulación 3 & 10.13 & $\pm 0.345^{a d}$ \\
\hline & Formulación 4 & 8.73 & $\pm 0.100^{\mathrm{ac}}$ \\
\hline & Formulación 5 & 8.74 & $\pm 0.133^{\mathrm{ac}}$ \\
\hline & $\begin{array}{c}\text { Formulación_6 (Gran } \\
\text { Señora (GS)) }\end{array}$ & 3.74 & $\pm 0.945^{\mathrm{ba}}$ \\
\hline & $\begin{array}{l}\text { Formulación_7 } \\
\text { (Fortiavena) }\end{array}$ & 4.46 & $\pm 0.095^{b d}$ \\
\hline & $\begin{array}{c}\text { Formulación_8 } \\
\text { (Quaker) }\end{array}$ & 4.32 & $0.302^{b d}$ \\
\hline
\end{tabular}

Letras iguales no existen diferencias estadísticamente significativas

De la tabla anterior se infiere que la humedad de las formulaciones 6,7 y 8 de avena instantánea difieren de las demás formulaciones $\mathrm{y}$ en todos los casos los valores fueron menores al máximo permitido para harina de avena (NTC 2160), así mismo la humedad se relaciona positivamente con el tamaño de la partícula, donde Quaker es la formulación que presenta menor humedad, tamaño de partícula, $\mathrm{CH}$ e ISA, por su parte la formulación 3 es la que presenta mayor humedad y tamaño de partícula evidenciado en su aglomeración, aunque estos parámetros no se ven evidenciados en la $\mathrm{CH}$ e ISA comparada con las demás formulaciones de la empresa.

Tabla 10. Prueba Post Hoc de Tukey de humedad para crema de arroz sabor arequipe.

\begin{tabular}{|c|c|cc|}
\hline $\begin{array}{c}\text { Parámetro } \\
\text { de calidad }\end{array}$ & MUESTRAS & \multicolumn{2}{|c|}{$\begin{array}{c}\text { MEDIA } \pm \\
\text { DESVIACION } \\
\text { ESTANDAR }\end{array}$} \\
\hline \multirow{4}{*}{ Humedad } & Formulacion_1 & 12.12 & $\pm 0.61^{\mathrm{a}}$ \\
\cline { 2 - 4 } & Formulacion_2 & 11.21 & $\pm 0.38^{\mathrm{a}}$ \\
\cline { 2 - 4 } & Formulacion_3 & 12.32 & $\pm 0.21^{\mathrm{a}}$ \\
\cline { 2 - 4 } & Formulacion_4 & 11.01 & $\pm 0.09^{\mathrm{b}}$ \\
\cline { 2 - 4 } & Formulacion_5 & 10.34 & $\pm 0.36^{\mathrm{b}}$ \\
\cline { 2 - 4 } & Formulación 6 & 6.74 & $\pm 0.38^{\mathrm{c}}$ \\
& (Arroz_Poli) & & \\
\hline
\end{tabular}

Letras iguales no existen diferencias estadísticamente significativas

De la tabla 10 se determinó que la humedad de la formulación 6 difiere de todas las demás formulaciones, así como la formulación 3 difiere de las formulaciones 4 , 5 y 6. Por otra parte el contenido de humedad de las harinas de las formulaciones 1 y 3 superaron la humedad máxima permitida para cereales (12 \%), posiblemente por la falta de trazabilidad en la materia prima y mal manejo en el almacenamiento manejado en la empresa.

\section{Prueba de Sedimentación.}

En la figura 6 se muestran los resultados de la prueba de sedimentación para avena instantánea sabor fresa tomando como patrón 2 muestras comerciales (Fortiavena y Quaker). Como se observa en la figura 6 las formulaciones patrón presentaron menor volumen y tiempo de sedimentación en comparación con las muestras elaboradas en la empresa, esto se debe a los tratamientos tecnológicos, ya que en la empresa no se hace un proceso de precocido de la materia prima, y este facilita 
@LIMENTECH CIENCIA Y TECNOLOGÍA ALIMENTARIA ISSN 1692-7125. Volumen 13, No. 2, p. 205-220, año 2015 Facultad de Ingenierías y Arquitectura Universidad de Pamplona

la solubilidad y retarda el tiempo de sedimentación

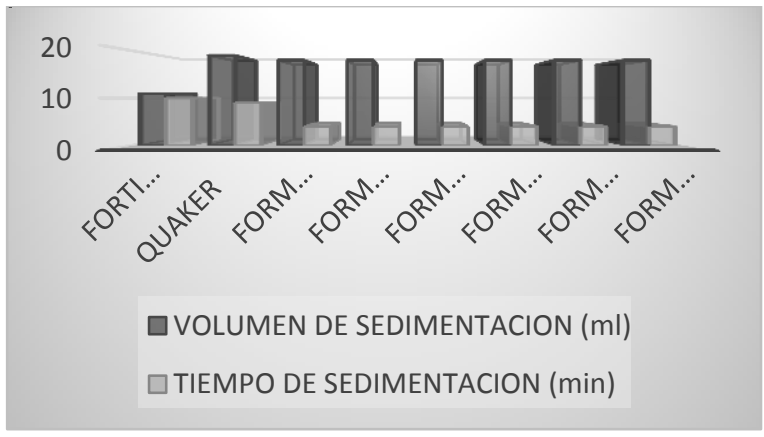

Figura 6. Prueba de sedimentación para Avena Instantánea sabor fresa.

Otro factor influyente es el tamaño de la partícula y su área superficial como ocurre con la avena Quaker, además la composición de los diferentes tipos de avena y la adición de estabilizantes presentan una respuesta positiva en la prueba de sedimentación ya que tienen como función impedir el cambio de forma o naturaleza química de los alimentos, inhibiendo reacciones o manteniendo el equilibrio químico de los mismos. (Biermann y Grosch, 1979).

\section{CONCLUSIONES}

Las pruebas fisicoquímicas determinaron que la calidad de las diferentes formulaciones evaluadas de avena instantánea y crema de arroz son similares a la calidad de las muestras patrón Fortiavena, Quaker y Arroz Poli, cumpliendo con los requisitos exigidos para estos productos.
En la figura 7 se muestra la prueba de sedimentación realizada a Crema de Arroz sabor arequipe elaborada por la empresa y un producto de la competencia.

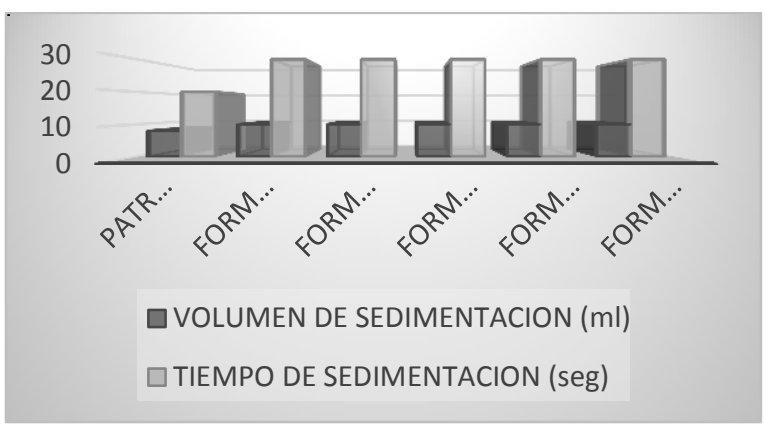

Figura 7. Prueba de sedimentación para Crema de Arroz.

La prueba de sedimentación aplicada a crema de arroz evidencia que el volumen de sedimentación de todas las muestras es muy similar, la diferencia es el tiempo de sedimentación, que es más corto en el producto de la competencia comparado con el de las formulaciones evaluadas. Por otra parte se ve claramente la diferencia entre el volumen y el tiempo de sedimentación en ambos productos, el cual es mayor en avena instantánea.

El precocido realizado a las materias primas influye significativamente en la calidad final de la avena instantánea y la crema de arroz, mejoran la $\mathrm{CH}$ y ISA adicionalmente influye en la dureza del grano antes de la molienda. Las formulaciones óptimas para avena instantánea sabor fresa fueron la formulación 2, y la formulación 3 y para crema de arroz fue la formulación 5 las 
cuales presentaron los mejores resultados en los análisis fisicoquímicos (porcentaje de cenizas, $\mathrm{CH}$, ISA, tamaño de partícula, humedad y volumen de sedimentación.

\section{REFERENCIAS BIBLIOGRÁFICAS}

Anderson, R. A., Conway, H. F., Pfeifer, V. F. and Griffin, E. L. (1969). Gelatinization of corn grits by roll and extrusion cooking. CerealSci. Today 14: 4, 11, 12.

Biermann U, and Grosch W. (1979). Bitter tasting monoglycerides from stored oat flour. Zeitschriftfür Lebensmitte luntersuchung und -Forschung $A$, 169:22-26.

Champagne, E. T., (2004). Rice: Chemistry and Technology. $3^{a}$ ed. St Paul MN: American Association of Cereal Chemists. Pág. 639.

Fan, J. and Marks B. P. (1998). Retrogradation kinetics of rice flours as influenced by cultivar. Cereal Chemistry, 75:153-155.

Fitzgerald, M. (2004). Starch. In: Champagne ET, editor. Rice: Chemistry and Technology, 3rd ed. St Paul MN: American Association of Cereal Chemists, Inc. Pág. 109 - 41.

Flores-Farías R., Martínez-Bustos F., Salinas-Moreno, Y., y Ríos, E. (2002) Caracterización de Harinas comerciales de maíz. Agrociencia, vol. 36, núm. 5, septiembre-octubre, pp. 557-567. Colegio de Postgraduados. Texcoco, México.

Gómez, M. H., Waniska, R. D. and Rooney, L. W. (1991). Starch characterization of nixtamalized corn flour. Cereal Chem. 68: 578-582.

Gómez, M., Oliete B, Caballero P. A., Ronda F, y Blanco C. A. Estudio de las características reológicas de las masas enriquecidas con harina de castaña. Actas del Congreso Internacional de Ciencia y Tecnología de los Alimentos, 272. Córdoba. Argentina. 15- 17 Noviembre de 2006.

Instituto Colombiano de Normas Técnicas y Certificación. ICONTEC.

(2006)

Productos de Molinería. Harina de Avena Precocida para consumo humano. Bogotá. (NTC 2160).

Instituto Colombiano de Normas Técnicas y Certificación. ICONTEC.

(2006)

Alimentos para Animales. Productos y Subproductos del Arroz. Bogotá. (NTC 476).

Juliano, B. O. (1984). The chemical basis of rice grain quality. En Proceedings of the 
Workshop on Chemical Aspects of Rice

Grain Quality, International Rice

Research Institute, Manila, Philippines.

Pág.68-89.

Leach, W. H., Mccowen, L. D. and Schoch,

T. J. (1959). Structure of starch granule;

I. Swelling and solubility patterns of various starches. Cereal Chemistry 36:534-544.

León, JJL. y Carreres, R. (2002). Calidad del arroz: Criterios para una adecuada valoración. Vida Rural, 145:38-40.

Normand, F. L., and Marshall, W. E. (1989).

Differential scanning calorimetry of whole grain milled rice and milled rice flour. Cereal Chemistry, 66:317-320.

Rosell, CM. (2007). Vitamin and mineral fortification of bread. En: Technology of Functional Cereal Products. Ed B. Hamaker. Woodhead Publishing Ltd, Cambridge, UK.

Saif, S. M. H., Lan, Y., and Sweat, V. E. (2003). Gelatinization properties of rice flour. International Journal of Food Properties, 6:531-542.

Sánchez, B. (2005). Caracterización fisicoquímica y funcional de la fibra dietética del fruto de níspero (Eriobotryajaponica) y de la cáscara de mango obo (Mangifera indica $L$ ). Tesis
Universidad Tecnológica de la Mixteca. Huajuapan de León, Oax, México.

Singh, V., Okadome, H., Toyoshima, H., Isobe, S., and Ohtsubo, K. (2000). Thermal and physicochemical properties of rice grain, flour and starch. Journal of griculture and Food Chemistry, 48:26392647. 